\title{
Resonance Fluorescence from Semiconductor Quantum Dots: Beyond the Mollow Triplet
}

Lund, Anders Mølbjerg; Nielsen, Per Kær; Lorke, Michael; Mørk, Jesper

Published in:

Physical Review Letters

Link to article, DOI:

10.1103/PhysRevLett.108.017401

Publication date:

2012

Document Version

Publisher's PDF, also known as Version of record

Link back to DTU Orbit

Citation (APA):

Lund, A. M., Nielsen, P. K., Lorke, M., \& Mørk, J. (2012). Resonance Fluorescence from Semiconductor Quantum Dots: Beyond the Mollow Triplet. Physical Review Letters, 108(1), 017401.

https://doi.org/10.1103/PhysRevLett.108.017401

\section{General rights}

Copyright and moral rights for the publications made accessible in the public portal are retained by the authors and/or other copyright owners and it is a condition of accessing publications that users recognise and abide by the legal requirements associated with these rights.

- Users may download and print one copy of any publication from the public portal for the purpose of private study or research.

- You may not further distribute the material or use it for any profit-making activity or commercial gain

- You may freely distribute the URL identifying the publication in the public portal 


\title{
Resonance Fluorescence from Semiconductor Quantum Dots: Beyond the Mollow Triplet
}

\author{
Anders Moelbjerg, ${ }^{*}$ Per Kaer, Michael Lorke, and Jesper Mørk \\ DTU Fotonik, Department of Photonics Engineering, Technical University of Denmark, Building 343, 2800 Kgs. Lyngby, Denmark
} (Received 13 July 2011; published 3 January 2012)

\begin{abstract}
We show that the resonance fluorescence spectrum of a quantum dot excited by a strong optical pulse contains multiple peaks beyond those of the Mollow triplet. We show that as the area of the optical pulse is increased, new side peaks split off the central peak and shift in frequency. A simple analytical theory has been derived, which quantitatively accounts for the appearance and position of the peaks. This theory explains the physics responsible for the multiple peaks. By considering the time-dependent spectrum we demonstrate a time ordering of the side peaks, which is further evidence for the suggested physical explanation.
\end{abstract}

DOI: 10.1103/PhysRevLett.108.017401

The coherent manipulation of semiconductor quantum dots has attracted much interest recently [1-3]. This research is motivated by the proposal of using quantum dots as the basic building block of quantum information technology, e.g., as single photon emitters [4] or qubits for quantum computers [5]. In order to reduce decoherence effects, resonant optical excitation is often employed in experiments with semiconductor quantum dots. For an ideal two-level system, it is well known that strong resonant excitation with a continuous wave (cw) field results in a so-called resonance fluorescence (RF) spectrum containing three peaks, known as the Mollow triplet. This iconic spectral feature was first predicted by Mollow [6] and finds a simple physical explanation, when considering the allowed optical transitions between the dressed states of an atom under the influence of a cw optical field [7]. The effect has been demonstrated experimentally for atoms [8] and single molecules [9], and recently also for selfassembled semiconductor quantum dots $[10,11]$.

In this Letter we calculate the resonance fluorescence spectrum of a quantum dot under pulsed excitation. Naively, one might expect the spectrum to be merely broadened or smeared out due to the time-varying degree of atomic state dressing. In contrast, we find a very rich spectrum containing multiple peaks and a characteristic scenario by which the peaks emerge from the central peak at discrete levels of the field amplitude (or pulse width) and subsequently spread to higher frequencies. We present detailed numerical investigations of the phenomenon based on a master equation model and find that the effect should be experimentally accessible, even when considering the relatively high dephasing rates of quantum dots due to phonon scattering. We also present an analytical model, derived using the stationary phase approximation [12], that quantitatively accounts for the emergence and spectral position of the side peaks. Based on this, we suggest a simple physical interpretation of the phenomenon, which attributes the side peaks to interference between fields emitted at different temporal positions along the pulse.
PACS numbers: 78.67.Hc, 42.50.Ct, 42.50.Hz, 78.55.-m

This suggests a specific time ordering between the peaks, which we confirm by analyzing the time-dependent resonance florescence spectrum.

The existence of multiple peaks in the resonance fluorescence spectrum under pulsed excitation has been pointed out before for atomic systems [13-17], and recently multiple peaks have been demonstrated in the emission from microplasmas using very strong pulses tuned far from resonance $[18,19]$. We show that this phenomenon should be observable in semiconductor quantum dots. In addition we provide new insight to the underlying physics by deriving an analytical criterion for the emergence of side peaks as well as their frequency positions and by investigating the buildup of the side peaks in the timedependent spectrum. We are thus in a position to provide a simple and well-founded physical explanation for the phenomenon. In contrast to earlier investigations we have included pure dephasing caused by phonons in our theoretical model, and we are therefore not limited to atomic systems, but are able to provide results for semiconductor quantum dots. We note that for such systems, pulsed excitation is often employed exactly because of the limitations implied by dephasing.

We use the master equation method [20] to obtain the RF spectrum from a quantum dot excited by a pulse. The quantum dot has two levels, denoted $g$ and $e$ for the ground and first excited state, respectively. They are coupled by a semiclassical interaction Hamiltonian $H_{\text {int }}(t)$. In the interaction picture it reads

$$
H_{\text {int }}(t)=\frac{1}{2} \hbar \Omega(t)\left(\sigma_{+}+\sigma_{-}\right)
$$

where the dipole and rotating wave approximations have been used, and where $\sigma_{+}=|e\rangle\langle g|$ and $\sigma_{-}=|g\rangle\langle e|$ are raising and lowering operators for the two-level system. The Rabi frequency $\Omega(t)$ is related to the slowly varying external electrical field amplitude $E$ by $\Omega=\frac{\mu E}{\hbar}$, where $\mu$ is the electric dipole moment of the quantum dot [7]. For pulsed excitation, $E=E(t)$, the Rabi frequency becomes time dependent, $\Omega=\Omega(t)$. We assume that the central 
frequency of the pulse $\omega_{0}$ is tuned to the electronic transition $\omega_{e g}$.

To take the semiconductor environment into account we include pure dephasing, arising from the interaction with phonons [21]. Pure dephasing and spontaneous decay are included in the time evolution of the system by using the von Neumann master equation with Lindblad terms, which is [20]

$$
\frac{d \rho(t)}{d t}=\frac{1}{i \hbar}\left[H_{\mathrm{int}}(t), \rho(t)\right]-\mathcal{L}_{\gamma} \rho(t)-\mathcal{L}_{\gamma^{*}} \rho(t) .
$$

Here, $\rho(t)$ is the density matrix in the interaction picture and $\mathcal{L}_{\gamma} \rho=\frac{\gamma}{2}\left(\sigma_{+} \sigma_{-} \rho+\rho \sigma_{+} \sigma_{-}-2 \sigma_{-} \rho \sigma_{+}\right)$and $\mathcal{L}_{\gamma^{*}} \rho=\gamma^{*}(t)\left(\sigma_{+} \sigma_{-} \rho+\rho \sigma_{+} \sigma_{-}-2 \sigma_{+} \sigma_{-} \rho \sigma_{+} \sigma_{-}\right)$are Lindblad terms for spontaneous decay and pure dephasing, with rates $\gamma$ and $\gamma^{*}(t)$, respectively. The pure dephasing rate is composed of a background term $\gamma_{0}^{*}$ and a phonon dependent term $D(t)$. It is possible to show that the dominant contribution to the pure dephasing rate, originating from acoustic phonons, is given by $D(t)=$ $\frac{1}{2} \alpha \pi \Omega(t)^{3} e^{-\left[\Omega(t) / \omega_{c}\right]^{2}} \operatorname{coth}\left(\hbar \omega / 2 k_{B} T\right)$, [22], where it has been assumed that the quantum dot confinement potential is harmonic and spherically symmetric. The cutoff frequency $\omega_{c}$ depends on the dot size $d$ as $\omega_{c}=\sqrt{2} u / d$, and we use parameters corresponding to GaAs bulk phonons: $u=5110 \mathrm{~m} / \mathrm{s}$ and $\alpha=0.032 \mathrm{ps}^{2}$. The inset in Fig. 1(a) shows the pure dephasing rate for a $4.5 \mathrm{~nm}$ quantum dot at $4 \mathrm{~K}$. The coupling to acoustic phonons also gives rise to a renormalization of the Rabi frequency, which is only relevant for elevated temperatures. As we are investigating the low temperature regime, this effect has not been included.

The time-dependent RF spectrum is obtained as the time-integrated Fourier transform of the correlation function [23]

$$
S(\omega, t) \propto \operatorname{Re} \int_{-\infty}^{t} d t^{\prime} \int_{-\infty}^{t-t^{\prime}} d \tau G\left(t^{\prime}, \tau\right) e^{i\left(\omega-\omega_{e g}\right) \tau},
$$

where the correlation function is $G(t, \tau)=\left\langle\sigma_{+}(t)\right.$ $\left.\sigma_{-}(t+\tau)\right\rangle$, and is obtained using the quantum regression theorem [24]. The usual (time-independent) RF spectrum is obtained as $S(\omega)=\lim _{t \rightarrow \infty} S(\omega, t)$.

In our numerical model, the quantum dot is excited by a single Gaussian pulse from an external laser, and the RF is measured by a perfect spectrometer. The Rabi frequency is $\Omega(t)=\Omega_{p} \exp \left[-\left(t-t_{0}\right)^{2} / \tau^{2}\right]$, where $\Omega_{p}=\mu E\left(t_{0}\right) / \hbar$ is the peak Rabi frequency and $2 \tau$ is the width of the electric field envelope of the pulse at $1 / e$ of the amplitude $(\mathrm{FWHM} \approx 1.67 \tau)$.

In Fig. 1(a), the RF spectrum of a quantum dot excited by a Gaussian pulse is shown for a spontaneous decay rate of $\hbar \gamma=10 \mu \mathrm{eV}$ and no pure dephasing (solid line). In contrast to the cw case, where the RF spectrum consists of tree peaks at $\omega_{e g}$ and $\omega=\omega_{e g} \pm \Omega_{p}$, the so-called Mollow triplet, pulsed excitation gives rise to several side
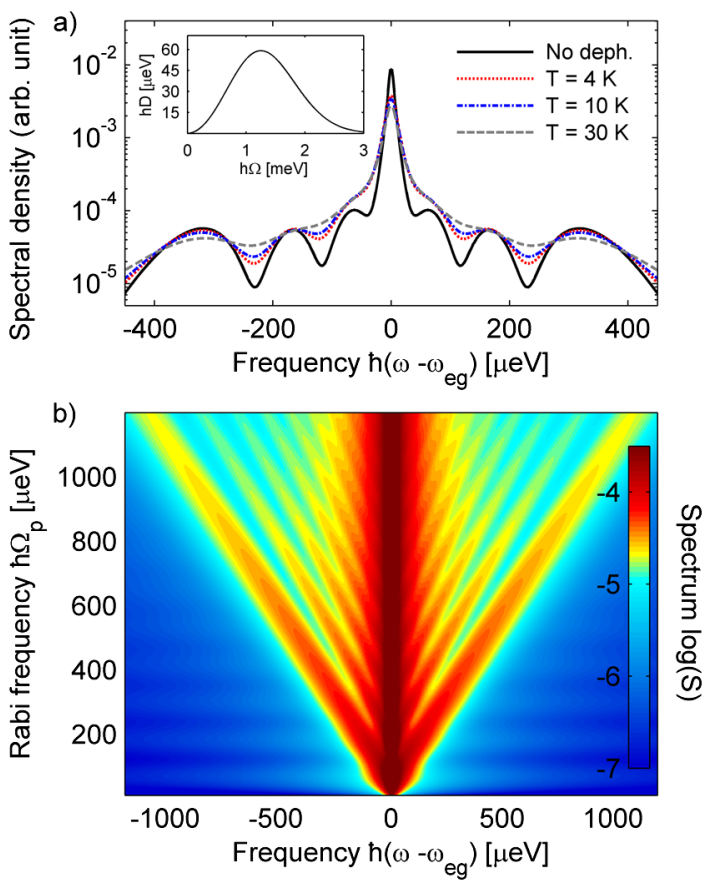

FIG. 1 (color online). (a) Calculated RF spectrum $S(\omega)$ for a quantum dot excited by a pulse with peak Rabi frequency, $\hbar \Omega_{p}=400 \mu \mathrm{eV}$ for the case of no pure dephasing (solid line) and for three different values of the temperature $(T=4,10$, and $30 \mathrm{~K})$. The inset shows the $\Omega$-dependent pure dephasing rate for $T=4 \mathrm{~K}$. (b) Contour plot of the RF spectrum as a function of the Rabi frequency $\Omega_{p}$ for $T=4 \mathrm{~K}$. In all cases, the pulse is Gaussian and has the width $2 \tau=40 \mathrm{ps}$, the spontaneous decay rate is $\hbar \gamma=10 \mu \mathrm{eV}$, the quantum dot size is $d=4.5 \mathrm{~nm}$, and the background dephasing rate is $\hbar \gamma_{0}^{*}=5 \mu \mathrm{eV}$.

peaks. These peaks are all closer to the central peak and lower in amplitude than the Mollow side peaks would be. The figure also shows the effect of phonon induced dephasing for different temperatures. It is apparent that the effect of the phonon coupling is to broaden and smear the spectral features, but the multiple peaks remain observable.

In Fig. 1(b) a contour plot of the RF emission is shown versus frequency and Rabi frequency for a background pure dephasing rate of $\hbar \gamma_{0}^{*}=5 \mu \mathrm{eV}$ and a temperature of $4 \mathrm{~K}$. As seen, for large Rabi frequencies the RF spectrum contains many side peaks. Peaks emerge from the central resonance frequency at certain specific values of the peak Rabi frequency and move outwards as the Rabi frequency is increased. This multipeak behavior points to physics beyond the conventional dressed state description.

It has recently been shown that Rabi oscillations [25] and emission from quantum dots $[3,26]$ are affected by dephasing originating from the excitation (EID). This $\Omega$-dependent dephasing can be attributed to the coupling to phonons, and the phenomenon is accounted for in the model presented above. Experimentally, this EID has proven to be large, and thus could destroy the multipeak structure of the spectrum. As shown in Fig. 1(b), where 
realistic values of the dot size and temperature have been used, EID does not hinder the detection of the multiple side peaks, however, the visibility of the side peaks decreases for large values of the Rabi frequency, which means that our theory can be used to identify optimized conditions for experimentally observing the phenomenon.

The RF spectrum is very dependent on the pulse width, as seen in Fig. 2. As the pulse width is increased, new peaks emerge and they are shifted outwards towards the position of the Mollow side peaks at $\omega=\omega_{e g} \pm \Omega_{p}$ (marked with two arrows above the figure). In the long pulse limit all the peaks merge together to form two large side peaks, and the Mollow result is recovered. New peaks emerge at discrete values of the pulse width in the same way that new peaks emerge for increasing Rabi frequency. This indicates that the number of peaks is dependent on the pulse area, which is proportional to $\Omega_{p} \tau$.

We have derived an approximate analytical model, which is found to quantitatively account for the emergence and spectral positions of the peaks in the RF spectrum. In addition, the model provides a good insight into the physics of the multiple peaks.

In the absence of any radiative decay and pure dephasing, the master equation can readily be solved in closed form and the off-diagonal elements of the density matrix, in the case where the quantum dot is initially in the ground state, are $\rho_{g e}(t)=\rho_{e g}^{*}(t)=\frac{i}{2} \sin [\phi(t)]$, where $\phi(t)=\int_{-\infty}^{t} \Omega\left(t^{\prime}\right) d t^{\prime}$

The coherent part of the RF spectrum [7] of the emitter can be found from the off-diagonal elements as

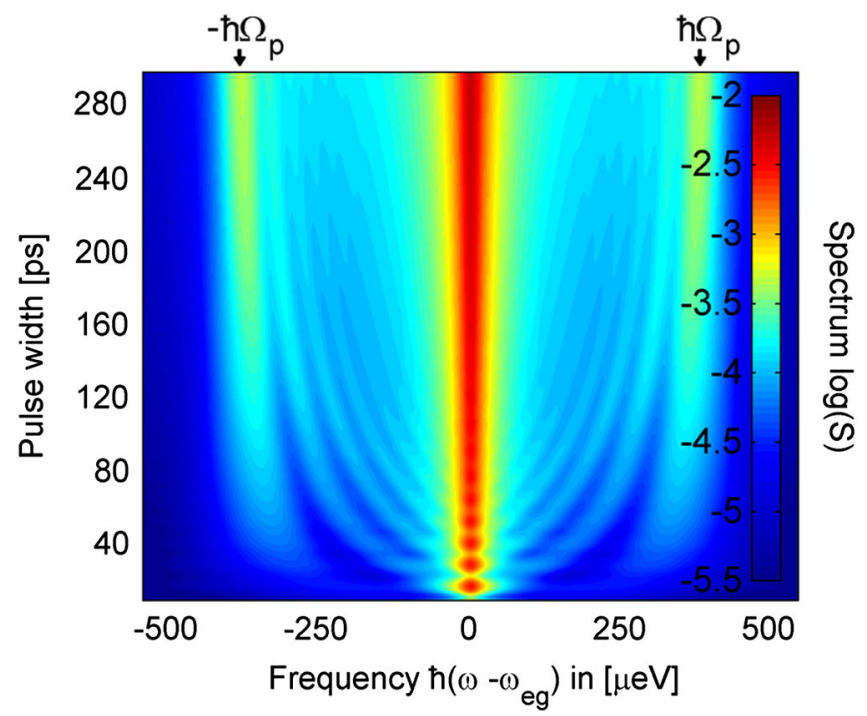

FIG. 2 (color online). Contour plot of the RF spectrum as a function of pulse width for a peak Rabi frequency of $\hbar \Omega_{p}=$ $400 \mu \mathrm{eV}$. The positions of the two side peaks, which would be obtained in the cw case, are shown with arrows at the top of the figure. The pulse is Gaussian and the spontaneous decay and pure dephasing rates are $\hbar \gamma=\hbar \gamma_{0}^{*}=10 \mu \mathrm{eV}$ and $D=0 \mu \mathrm{eV}$.

$$
S(\omega) \propto\left|\int_{-\infty}^{\infty} \sin [\phi(t)] e^{i\left(\omega-\omega_{e g}\right) t} d t\right|^{2} .
$$

Using the numerical model we have shown that, in contrast to the Mollow case [20], the coherent part of the RF spectrum will contain side peaks.

The stationary phase approximation [12] can be used to evaluate the integral in Eq. (4), and to obtain a simple criterion for the emergence and positions of side peaks. The criterion can be expressed as

$$
\phi(t)-\phi(-t)-2 \Omega(t) t=\left(2 n+\frac{1}{2}\right) \pi,
$$

where $n$ is an integer or zero. The solutions to Eq. (5), labeled $t_{n}$, are unique temporal positions along the pulse. The frequency of the emission at that temporal position is $\omega_{e g}+\Omega\left(t_{n}\right)$ and corresponds to one of the peaks in the spectrum. For resonant excitation the spectrum is symmetric, and another peak is located at $\omega_{e g}-\Omega\left(t_{n}\right)$.

From this criterion it can immediately be seen that, as $t=0$ is not a solution, there can be no peaks exactly at $\omega=\omega_{e g} \pm \Omega_{p}$, i.e., the "classical" position of the Mollow side peaks.

For any symmetrical pulse envelope, the third term on the left-hand side of Eq. (5) vanishes and the two first terms become the pulse area, $\Theta=\phi(\infty)$, for $t \rightarrow \infty$. Since the left-hand side of Eq. (5) is an increasing function of $t$, new peaks in the RF spectrum will appear when $\Theta>\left(2 n+\frac{1}{2}\right) \pi$. Because $\Omega(t) \rightarrow 0$ for $t \rightarrow \infty$, these peaks start out at $\omega=\omega_{\text {eg }}$. This is in good agreement with the qualitative behavior seen in Fig. 1(b). For a Gaussian envelope the pulse area is $\Omega_{p} \tau \sqrt{\pi}$. Referring to Fig. 1(a) where the pulse area is $\Omega_{p} \tau \sqrt{\pi}=400 \mu \mathrm{eV} \times 20 \mathrm{ps} \sqrt{\pi} / \hbar=21.5$, this model indicates that the RF spectrum should contain 4 peaks, since $\left(2 \times 3+\frac{1}{2}\right) \pi=20.4$. However, the innermost peak with $n=3$ is at $\hbar \omega=\hbar \omega_{e g} \pm 8.1 \mu \mathrm{eV}$, and is therefore hidden by the broad central peak. This leaves the three peaks which are visible in the figure.

Figures 3(a) and 3(b) compare the peak positions predicted by this analytical model with the numerical model discussed in the previous sections. The figures show the positions of the side peaks for variable Rabi frequency and constant pulse width [3(a)] and for constant Rabi frequency and variable pulse width [3(b)], corresponding to the results shown in Figs. 1 and 2 respectively. The analytical theory is seen to be in very good quantitative agreement with the full numerical solutions.

The physics captured by the stationary phase approximation is that a peak in the RF spectrum occurs as a result of constructive interference of the emission belonging to two different parts of the pulse, namely, those temporal positions $t_{n}$ that correspond to the same instantaneous frequency $d \phi /\left.d t\right|_{t=t_{n}}=\Omega\left(t_{n}\right)$ and where the field components add in phase. Each side peak in the RF spectrum can be related to the side peak of a Mollow triplet, with a Rabi frequency $\Omega\left(t_{n}\right)$. The varying envelope of the pulse means 
a)

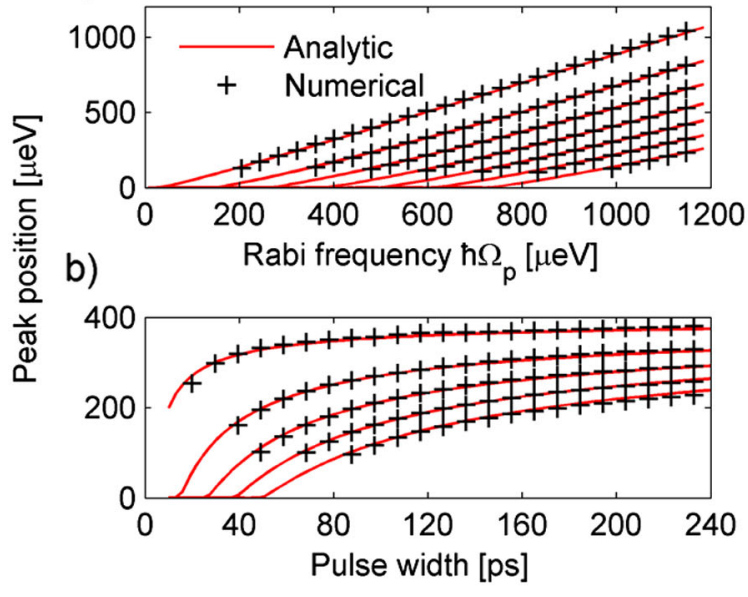

c)

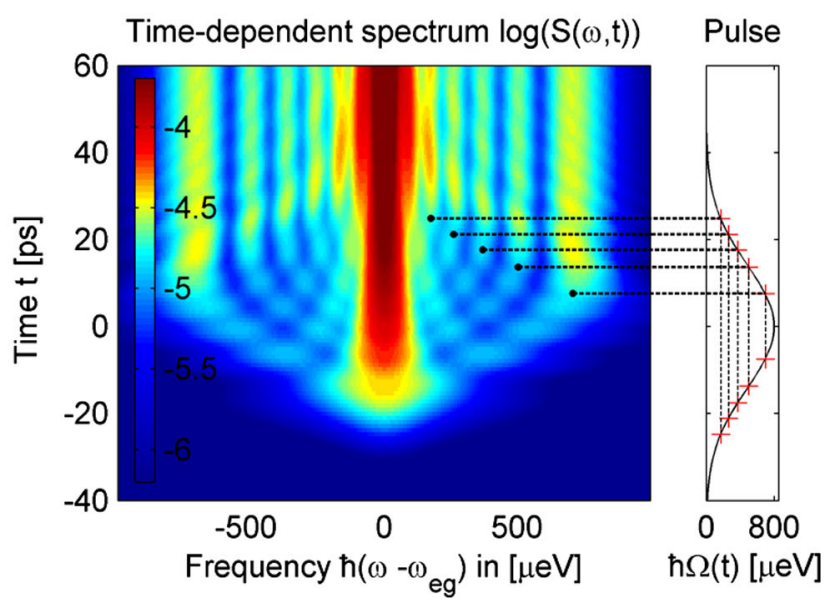

FIG. 3 (color online). (a),(b) The spectral position of the peaks in the RF spectra as found with the criterion in Eq. (5) (solid lines) compared with the results of the numerical model as shown in Figs. 1(b) and 2, respectively. (c) A contour plot of the time-dependent RF spectrum as a function of time and frequency. Also shown is the time-dependent Rabi frequency $\Omega(t)$. The stationary phase points are indicated with marks and connected with vertical lines. Black dots indicate the peak positions as found by the analytical model. The pulse is Gaussian and has a width of $2 \tau=40 \mathrm{ps}$, the spontaneous decay and pure dephasing rates are $\hbar \gamma=\hbar \gamma_{0}^{*}=10 \mu \mathrm{eV}$ and $D=0 \mu \mathrm{eV}$. The peak Rabi frequency is $\hbar \Omega_{p}=800 \mu \mathrm{eV}$.

that it gives rise to a continuum of Rabi frequencies, but because of interference, only a few of these will result in side peaks. A similar phenomenon is seen in optical fibers, where a propagating pulse undergoes self-phasemodulation, leading to multiple peaks in the spectrum [27]. Very recent work, [18,19], has suggested a similar physical explanation of the effect seen in microplasmas, and also proposed a simple analytical model for the appearance of points of destructive interference. However, our work extends that model to general pulse shapes and, as shown below, we find that the time-dependent spectrum gives additional insight into the time development of the peaks.

So far we have shown and explained the spectrum in the long time limit, which is the RF spectrum that would be observed in a time-integrated measurement. The behavior may be further investigated by considering the timedependent spectrum. In Fig. 3(c) the time-dependent RF spectrum, defined as in [23], is shown as a contour plot for a Rabi frequency of $\hbar \Omega_{p}=800 \mu \mathrm{eV}$. Also shown in the figure is the time-dependent Rabi frequency $\Omega(t)$. The figure shows that a single spectral peak emerges in the leading edge of the pulse. This peak then splits off two additional peaks, which are shifted outwards as time progresses. The side peaks frequency shift until, at a certain time, they appear to lock at a final position and increase in strength. The next set of side peaks is then split off the central peak a bit later, and is locked at its final frequencies later than the outermost peaks. The peaks are locked at their final frequency positions at a specific time and then increase in amplitude. We thus find a specific time ordering of the side peaks in the spectrum.
Our analytical model suggests that the appearance of a peak in the RF spectrum is a result of the constructive interference of the emission at the stationary phase points $t_{n}$ and $-t_{n}$ along the pulse. The "later" stationary phase points, $t_{n}$, are indicated by vertical lines on the pulse (temporal Rabi frequency) profile also shown in Fig. 3(c). It is clearly seen that these agree very well with the temporal positions at which the side peaks gain strength. Our model thus explains the observed time ordering: In order for a peak to have fully developed in the RF spectrum, the pulse must have evolved in time to include both $-t_{n}$ and $t_{n}$. So at time $t=t_{n}$ the frequency components required to produce the $n$th peak are present, and the peak is locked in place and increase in amplitude.

In conclusion, we have identified and explained the characteristic spectral features of the emission from a quantum dot excited by a strong pulse, which we believe have been previously overlooked. The resonance fluorescence spectrum thus exhibits multiple peaks, beyond those of the "classical" cw Mollow triplet. We have derived a simple analytical model that quantitatively accounts for the conditions under which the side peaks appear, as well as their tuning properties upon variation of the excitation conditions. The phenomenon can be interpreted as a result of interference between emission at different temporal positions along the pulse, an explanation we have further confirmed by considering the timedependent spectrum. We believe this effect should be experimentally observable in semiconductor quantum dots, and that it will provide another tool for characterizing such structures. 
*amolu@ fotonik.dtu.dk

[1] A. J. Ramsay, Semicond. Sci. Technol. 25, 103001 (2010).

[2] A. Muller, E. B. Flagg, P. Bianucci, X. Y. Wang, D. G. Deppe, W. Ma, J. Zhang, G. J. Salamo, M. Xiao, and C. K. Shih, Phys. Rev. Lett. 99, 187402 (2007).

[3] S. M. Ulrich, S. Ates, S. Reitzenstein, A. Löffler, A. Forchel, and P. Michler, Phys. Rev. Lett. 106, 247402 (2011).

[4] P. Michler, A. Imamoglu, M. D. Mason, P. J. Carson, G. F. Strouse, and S. K. Buratto, Nature (London) 406, 968 (2000).

[5] D. Loss and D. P. DiVincenzo, Phys. Rev. A 57, 120 (1998).

[6] B. R. Mollow, Phys. Rev. 188, 1969 (1969).

[7] C. Cohen-Tannoudji, J. Dupont-Roc, and G. Grynberg, Atom-Photon Interactions (Wiley-VCH, Weinheim, 2004).

[8] F. Schuda, C. R. Stroud, Jr, and M. Hercher, J. Phys. B 7, L198 (1974).

[9] G. Wrigge, I. Gerhardt, J. Hwang, G. Zumofen, and V. Sandoghdar, Nature Phys. 4, 60 (2007).

[10] E. B. Flagg, A. Muller, J. W. Robertson, S. Founta, D. G. Deppe, M. Xiao, W. Ma, G. J. Salamo, and C. K. Shih, Nature Phys. 5, 203 (2009).

[11] A. N. Vamivakas, Y. Zhao, C.-Y. Lu, and M. Atatüre, Nature Phys. 5, 198 (2009).

[12] E. T. Copson, Asymptotic Expansions (Cambridge University Press, Cambridge, England, 1965).
[13] P. Rodgers and S. Swain, Opt. Commun. 81, 291 (1991).

[14] L. F. Roberto Buffa, Stefano Cavalieri, and M. Matera, J. Phys. B 21, 239 (1988).

[15] K. Rza̧żewski and M. Florjańczyk, J. Phys. B 17, L509 (1984).

[16] M. Lewenstein, J. Zakrzewski, and K. Rzążewski, J. Opt. Soc. Am. B 3, 22 (1986).

[17] M. Florjańczyk, K. Rzążewski, and J. Zakrzewski, Phys. Rev. A 31, 1558 (1985).

[18] R. Compton, A. Filin, D. A. Romanov, and R. J. Levis, Phys. Rev. Lett. 103, 205001 (2009).

[19] R. Compton, A. Filin, D. A. Romanov, and R. J. Levis, Phys. Rev. A 83, 053423 (2011).

[20] H. J. Carmichael, Statistical Methods in Quantum Optics (Springer, New York, 1999), Vol. 1.

[21] P. Kaer, T. R. Nielsen, P. Lodahl, A.-P. Jauho, and J. Mørk, Phys. Rev. Lett. 104, 157401 (2010).

[22] A. Nazir, Phys. Rev. B 78, 153309 (2008).

[23] J. H. Eberly, C. V. Kunasz, and K. Wodkiewicz, J. Phys. B 13, 217 (1980).

[24] M. Lax, Phys. Rev. 129, 2342 (1963).

[25] A. J. Ramsay, T. M. Godden, S. J. Boyle, E. M. Gauger, A. Nazir, B. W. Lovett, A. M. Fox, and M. S. Skolnick, Phys. Rev. Lett. 105, 177402 (2010).

[26] C. Roy and S. Hughes, Phys. Rev. Lett. 106, 247403 (2011).

[27] G. P. Agrawal, Nonlinear Fiber Optics (Academic, New York, 2007), 4th ed. 